\title{
A!
}

This is an electronic reprint of the original article.

This reprint may differ from the original in pagination and typographic detail.

Mäkelä, Mikko; Geladi, Paul; Rissanen, Marja; Rautkari, Lauri; Dahl, Olli

\section{Hyperspectral near infrared image calibration and regression}

Published in:

Analytica Chimica Acta

DOI:

10.1016/j.aca.2020.01.019

Published: 08/04/2020

Document Version

Peer reviewed version

Published under the following license:

CC BY-NC-ND

Please cite the original version:

Mäkelä, M., Geladi, P., Rissanen, M., Rautkari, L., \& Dahl, O. (2020). Hyperspectral near infrared image calibration and regression. Analytica Chimica Acta, 1105, 56-63. https://doi.org/10.1016/j.aca.2020.01.019

This material is protected by copyright and other intellectual property rights, and duplication or sale of all or part of any of the repository collections is not permitted, except that material may be duplicated by you for your research use or educational purposes in electronic or print form. You must obtain permission for any other use. Electronic or print copies may not be offered, whether for sale or otherwise to anyone who is not an authorised user. 


\title{
Hyperspectral near infrared image calibration and regression
}

\author{
Mikko Mäkeläa,b*, Paul Geladib, Marja Rissanen ${ }^{\mathrm{a}}$, Lauri Rautkari ${ }^{\mathrm{a}}, \mathrm{Olli}^{\mathrm{b}} \mathrm{Dahl}^{\mathrm{a}}$
}

aAalto University, School of Chemical Engineering, Department of Bioproducts and Biosystems, PO Box 16300, 00076 Aalto, Finland

${ }^{\mathrm{b}}$ Swedish University of Agricultural Sciences, Department of Forest Biomaterials and Technology, Skogsmarksgränd, 90183 Umeå, Sweden

E-mail: mikko.makela@aalto.fi,mikko.makela@slu.se (M. Mäkelä) 


\begin{abstract}
Reference materials are used in diffuse reflectance imaging for transforming the digitized camera signal into reflectance and absorbance units for subsequent interpretation. Traditional white and dark reference signals are generally used for calculating reflectance or absorbance, but these can be supplemented with additional reflectance targets to improve the accuracy of reflectance transformations. In this work we provide an overview of hyperspectral image regression and assess the effects of reflectance calibration on hyperspectral image regression using partial least squares. Linear and quadratic reflectance transformations based on additional reflectance targets decrease average measurement errors and make it easier to estimate model pseudorank during image regression. The lowest measurement and prediction errors were obtained with the column and wavelength specific quadratic transformations which retained the spatial information provided by the line-scanning instrument and reduced errors in predicted concentration maps.
\end{abstract}

Keywords: hyperspectral imaging, reflectance calibration, prediction, partial least squares, textile analysis, pseudorank 


\section{Introduction}

Hyperspectral imaging combines the spatial information of an image with the chemical information of a spectrum and is finding more and more applications in various fields of research and process monitoring. Hyperspectral images are three-way datasets, where two ways or modes represent the spatial attributes of an image and the third one the spectral variables. Each pixel thus contains a spectrum which has been generated by the interaction of incident light with the approximate pixel area of the sample. The pixel spectra represent a type of chemical fingerprints, which can be used for e.g. classifying different areas of an image or for determining the spatial differences in analyte concentrations through hyperspectral image regression. Although hyperspectral imaging was originally developed within the remote sensing community $[1,2]$, it has spread rapidly to agriculture [3], pharmaceuticals [4], medical diagnostics [5], textiles [6] and materials science in general [7].

Hyperspectral imaging in the near infrared (NIR) region is based on measuring diffuse reflectance or transmission of polychromatic light. Chemometric interpretation of NIR spectra is however usually based on absorbance units to conform with the Beer-Lambert law that enables determining analyte concentrations based on the absorbance of light $[8,9]$. In diffuse reflectance imaging a sample is illuminated and the reflected light is measured by the camera detector. Transformation of the digitized raw image requires the use of at least one reference material for translating the camera signal into reflectance and absorbance units. A common alternative, recommended by most manufacturers, is to use a Spectralon white reference for measuring maximum reflectance and a dark current reading that has been obtained by covering the camera objective or using a shutter in front of the detector. Sample reflectance is then calculated as a portion of the maximum reflectance after the background dark current has been subtracted. Although this transformation is generally called one-point calibration, it is mathematically a two-point linear calibration procedure based on 
measured extreme values and provides no indication of potential non-linearities over the dynamic range of the instrument $[10,11]$.

The quality of hyperspectral imaging data is becoming increasingly important as the user community expands and the availability of images increases [12]. Results from image interpretation through chemometric classification and calibration models are also valid only as long as the sample, instrument and measurement environment remain stable [13]. Signal transformation and instrument drift issues can be reduced by using reference materials or targets in image calibration in the intensity, wavelength and spatial domains [14-16]. Previously Burger contributed significantly to the development of calibration procedures for hyperspectral NIR images through work included in his doctoral thesis published in 2006 [13]. As an example, the author discussed available reflectance and wavelength standards and developed procedures for image calibration [10] and diagnostics [17]. He used internal and external reflectance targets for image calibration and applied different transformation functions in the intensity domain to improve the quality of imaging data. A central question however remains unanswered; how does reflectance calibration affect the interpretation of hyperspectral images? This work attempts to answer that question in the context of hyperspectral image regression.

Here, we provide an overview of the principles of hyperspectral image regression and have followed the procedure adopted by Burger and Geladi [10] for transforming the detector signal into reflectance units using calibrated reflectance targets. In addition to the accuracy of different transforms, we determined the effect of reflectance calibration on image interpretation based on partial least squares (PLS) regression. An example image dataset was compiled from custom-made textile samples which were prepared using different ratios of cotton and polyester (PET) and subsequently imaged using a line-scanning instrument. The use of hyperspectral NIR cameras is a timely topic in textile analysis as it allows developing methods to classify or predict the properties of textiles for sorting [18] and finishing operations [19]. Overall, this work aims to continue the 
work on image calibration for improving the quality of hyperspectral imaging data and will help to clarify the effects of reflectance calibration on interpretation through hyperspectral image regression.

\section{Materials and methods}

\subsection{Sample preparation}

Textile samples were prepared for the imaging procedure by mixing cotton and PET strands and producing single jersey knits with horizontal stripes that contained different cotton and PET contents. Two separate samples with approximate dimensions of $80 \times 70 \mathrm{~mm}$ were produced for use as calibration and test sets. The calibration sample contained five approximately $10 \mathrm{~mm}$ stripes with $0 \%, 26.9 \%, 53.8 \%, 80.7 \%$ and $100 \%$ of PET on mass basis. The test sample contained three approximately $20 \mathrm{~mm}$ stripes with $0 \%, 53.8 \%$ and $100 \%$ of PET.

For the preparation of the textile samples, PET fibers with a fiber length of $38 \mathrm{~mm}$ (Indorama Corp.) were first carded with a carding machine (337A, Mesdan Lab) to obtain a sliver. The sliver was further elongated with a draw frame (Stiro Roving Lab 3371, Mesdan Lab) and formed into a false-twisted roving. The PET roving was ring spun (Ring Lab 82BA, SER.MA.TES Srl) into a strand which was mixed with a cotton strand (700 tpm, Gebrueder Otto GmbH \& Co.) to form the final yarn.

A total of four strands were used, which provided five different cotton and PET combinations including the pure components. The linear densities of the cotton and PET strands were measured with wrap reel (160 M, Mesdan Lab) and were 21.1 and 22.7 tex for cotton and PET, respectively.

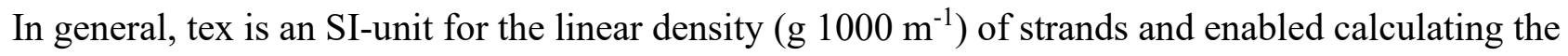
exact composition of different strand mixtures on a mass basis. The final samples were then 
prepared from the yarns of different strand combinations using a computer-programmed weft knitting machine (Stoll CMS ADF 32W E7.3 multigauge).

\subsection{Hyperspectral imaging}

The hyperspectral images were taken with a Specim SWIR 3 (Specim, Spectral Imaging, Ltd) hyperspectral camera [21]. The instrument was equipped with a $30 \mathrm{~mm}$ OLES lens provided by Specim (Spectral Imaging, Ltd) with a field of view of $54 \mathrm{~mm}$. The textile samples were imaged through quartz glass to minimize wrinkles and random light scattering. The camera operated in linescanning mode where a line of 384 pixels was continuously recorded within 935-2550 nm with a nominal spatial resolution of $0.14 \mathrm{~mm}$ per pixel. Two rows of quartz halogen lamps generated polychromatic light and the reflected wavelengths were separated by grating-prism monochromator followed by a HgCdTe detector array. Spectral sampling was every $5.6 \mathrm{~nm}$ which provided a total of 288 spectral variables with a reported spectral resolution of $12 \mathrm{~nm}$ (full width half maximum). The integration time was adjusted to approximately $2.8 \mathrm{~ms}$ so that the maximum white reference signal was lower than $90 \%$ of the maximum 16-bit signal output. A total of 25 dark reference images were taken with each sample image. In addition, images of 2, 25, 50, 75 and 99\% reflectance targets, which contained a decreasing amount of carbon black on a white Teflon base material, were also obtained. The reflectance targets were externally calibrated by Labsphere (Labsphere, Inc.) in an 8\% hemispherical setup within 250-2500 nm using a $50 \mathrm{~nm}$ interval. Individual images of each target were measured before the actual textile samples. The $50 \%$ target was imaged twice and was also used as a test set for testing the used reflectance transformations.

\subsection{Image calibration and transformation}

Regions of interest (ROIs) of $25 \times 384$ pixels were selected from the center of the reflectance targets for reflectance calibration. These spatial dimensions were chosen to enable a fair comparison with the common approach where 25 white and dark reference images are obtained for 
two-point calibration with this instrument. In addition, a ROI of $384 \times 384$ pixels was selected from the second image of the $50 \%$ target used as a reflectance test set. After segmentation, the raw target ROIs were median filtered with a moving window of $3 \times 3$ pixels to remove the effect of dead pixels in the camera detector. The "true" reflectance values of the targets were interpolated with a second order polynomial to obtain the necessary reference values that matched the wavelengths sampled by the hyperspectral camera, see Fig. A.1. Wavelengths outside 1000-2500 nm were excluded to remove unnecessary noise on extreme wavelengths. As illustrated in Fig. 1, two classes of signal transformations were determined for the $50 \%$ reflectance test set and the sample images. The global calibration models used wavelength dependent global mean values of the reference and target values and thus neglected the spatial dependencies of the camera detector. The column-wise models on the other hand enabled a position and wavelength dependent correction of image pixels separately for each image column, which preserved the spatial information provided by the instrument.

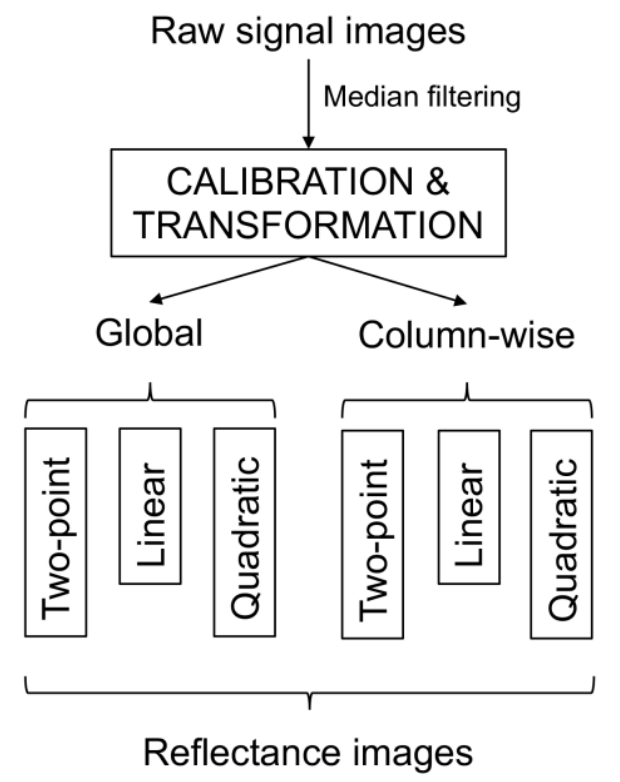

Fig. 1: Two classes of signal transformations were used in hyperspectral image calibration and transformation. 
Three different mathematical signal transformations were considered for both the global and column-wise model classes. The two-point linear transformations were based on the white and dark reference signals typically used in hyperspectral imaging according to Eq. (1) [10, 14]:

$r=(s-d r) \cdot(w r-d r)^{-1}$

where $r$ and s denote the wavelength specific unitless reflectance and raw signal values for each pixel and the $d r$ and $w r$ the respective dark and white reference signals. We deliberately avoid the use of symbol subscripts here for describing spatial and wavelength dependencies. A normalized version of the $99 \%$ reflectance target image was used as the white reference signal for all two-point transformations to eliminate the effect of different reference materials.

In addition, image ROIs of the 2, 25, 50, 75 and $99 \%$ reflectance targets with true calibrated values were used for obtaining more accurate reflectance transforms. This procedure involved fitting least squares regression models to the true reflectance versus measured average raw signal values for the used reflectance targets and then using the regression coefficients for transforming the $50 \%$ reflectance test set and the sample images. Two different regression models were considered. The models enabled a linear and a quadratic transformation to reflectance units according to Eqs. (2) and (3), respectively:

$r=b_{0}+b_{1} \cdot s$

$r=b_{0}+b_{1} \cdot s+b_{11} \cdot s^{2}$

where $b_{0}, b_{1}$ and $b_{11}$ describe the regression coefficients. The coefficients were obtained separately for each wavelength variable and for each image column for the column-wise transformations. No dark current subtraction was performed for the linear and quadratic transformations as this offset was included in the $b_{0}$ coefficient in Eqs. (2) and (3). Finally, the obtained reflectance images were converted to absorbance according to Eq. (4): 
$A=-\log _{10}(r)$

where $r$ describes the unitless reflectance values and $A$ the respective absorbance values in arbitrary units.

\subsection{Hyperspectral image regression}

Hyperspectral image regression enables predicting analyte concentrations in individual image pixels based on a multivariate calibration model $[22,23]$. This calibration procedure involves regressing typically externally determined or otherwise known sample properties on image spectra and should not be confused with the previously discussed image calibration against reflectance targets. The regression model equation in Eq. (5) illustrates the general relationship between analyte concentrations and a matrix of spectral objects:

$\mathbf{y}=\mathbf{X b}+\mathbf{e}$

where $\mathbf{y}$ denotes a $n \times 1$ vector of mean centered analyte concentrations, $\mathbf{X}$ a $n \times m$ matrix of $n$ mean centered calibration spectra on $m$ wavelengths, $\mathbf{b}$ a $m \times 1$ vector of regression model coefficients and e a $n \times 1$ vector of model residuals. As hyperspectral images contain significantly more pixel objects than wavelength variables, the $\mathbf{X}$ matrix typically consists of average spectra calculated from a set of calibration images. The determination of these average spectra is best performed after the three-way datasets have been reorganized into two-way arrays using an image unfolding operation that combines the spatial pixel modes. As illustrated in Fig. 2, this unfolding is generally performed by extracting slabs of image pixels in the column direction (Fig. 2A) and unfolding the slabs to form a section of the unfolded two-way array (Fig. 2B). 

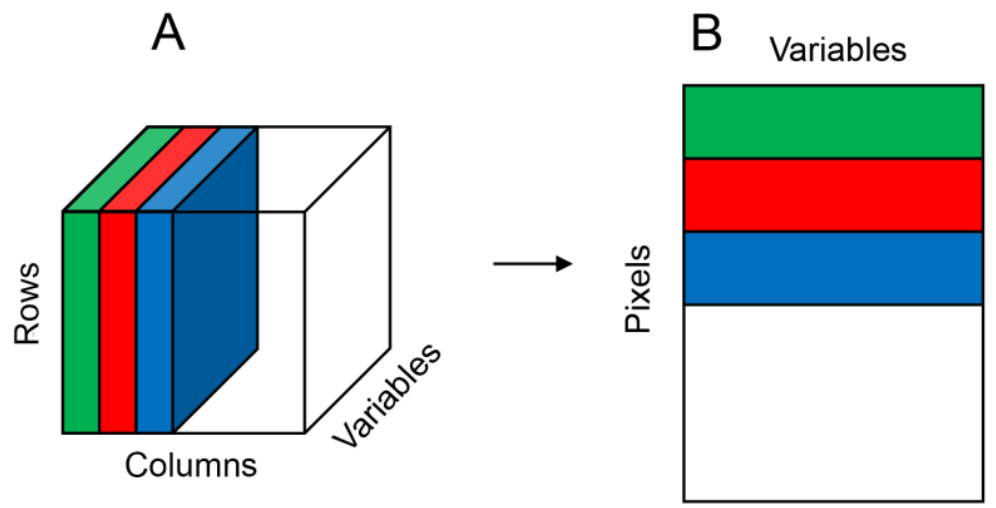

Fig. 2: Unfolding of a three-way image hypercube (A) into a two-way array (B).

Once the unfolding has been performed, the calculation of one or several average spectra is straightforward. The objective of multivariate calibration is to obtain a robust estimate of the model vector $\mathbf{b}$ in Eq. (5) which can then be used for predicting analyte concentrations in the individual image pixels according to Eq. (6):

$\widehat{\boldsymbol{y}}=\mathbf{Z b}$

where $\widehat{\boldsymbol{y}}$ denotes a $k \times 1$ vector of predicted pixel concentrations, $\mathbf{Z}$ a $k \times \mathrm{m}$ complete array of $k$ unfolded image spectra on $m$ wavelengths and $\mathbf{b}$ the regression vector from Eq. (5). The wavelength averages from mean centering $\mathbf{X}$ in Eq. (5) are used for mean centering $\mathbf{Z}$. The mean of the original $\mathbf{y}$ values also needs to be added to $\widehat{\boldsymbol{y}}$ and the predicted values can then be refolded back to the original image dimensions as a form of a concentration map.

There are several different ways to determine the model vector $\mathbf{b}$, which will not be discussed here for brevity. In this this work we used the PLS1 method based on the SIMPLS algorithm included in PLS Toolbox (Version 8.7, Eigenvector Research, Inc.) for Matlab® (Version 9.5, The MathWorks, Inc.) for predicting the PET contents of the textiles on a mass basis. PLS is the most common method for multivariate and image regression and takes into consideration the covariance structures of both the $\mathbf{y}$ and $\mathbf{X}$ blocks in Eq. (5). The interested reader should refer to the published literature 
for details on PLS, e.g. [24-26]. We extracted a total of 40 average spectra from the calibration sample with five different PET contents for model calibration. Eight spectra were calculated from each of the five chemically different areas in the sample based on equivalent ROIs of $50 \times 384$ pixels. In addition, a test set of 24 average spectra were extracted from the test sample with three $100 \times 384$ pixel ROIs using eight average spectra per each ROI. Raw signal images of the textile samples with the different ROIs used for extracting the calibration and test set spectra are illustrated in Fig. 3.
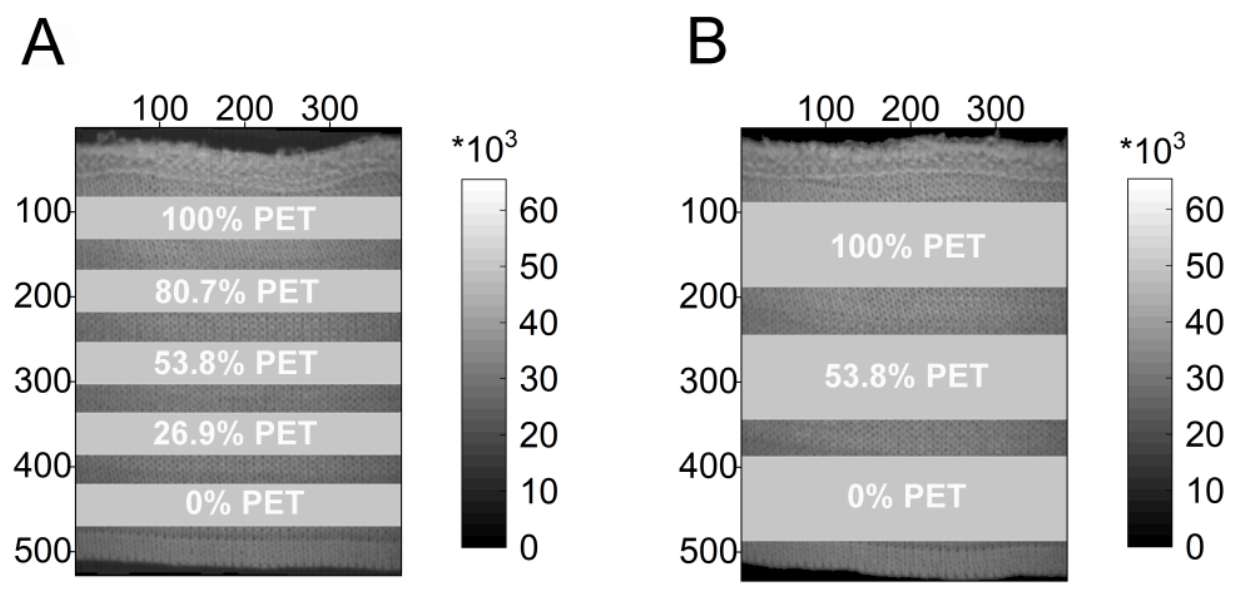

Fig. 3: Gray scale raw signal images of the calibration (A) and test (B) samples showing the selected ROIs and respective PET contents on a mass basis. The images were plotted based on the raw signal values on $1603 \mathrm{~nm}$. The axes denote pixel dimensions.

PLS model performance was evaluated based on the root mean squared errors (RMSE) of calibration (RMSEC) and prediction of the test set (RMSEP) as shown in Eq. (7):

$R M S E=\sqrt{\frac{\sum_{i=1}^{j}\left(y_{i}-\hat{y}_{i}\right)^{2}}{j}}$

where $j$ denotes the number of calibration or prediction objects. In addition, a RMSE parameter specifically derived from the predicted test set pixels, RMSEP Im, was determined. RMSEP ${ }_{\operatorname{Im}}$ was 
derived as in Eq. (7) by pooling the squared residuals of predicted pixel values and the known PET contents across the three test sample ROIs. Data analysis was performed using in-house Matlab® scripts, image segmentation was performed in Breeze (Version 2019.02, Prediktera AB) and the data were plotted in OriginPro (Version 9.6.0.172, Originlab Corp.).

\section{Results and discussion}

\subsection{Image calibration and transformation}

Reflectance transformations enable translating the raw camera signal into reflectance units. The accuracy of these transforms depends on the used transformation function and the number and range of the available reference or target signals. The typical two-point linear transformations based on white and dark references cover the entire signal range, but fail to consider potential nonlinearities in signal intensity $[10,16]$. Using several reflectance targets helps to counteract this issue. In addition, targets spread across the range of imaged samples help to minimize potential instability issues with the instrument during the imaging procedure. Fig. 4A illustrates the average normalized white reference, example dark current, and the reflectance target signals in the middle of the imaging plane. The average across the entire wavelength range are given in Fig. A.2. As shown in Fig. 4A, we found only small deviations from linearity over the dynamic range of the instrument, which mainly became visible with increasing signal intensity towards the centre of the wavelength range.

In addition to the two-point linear transformation, we fitted first and second order regression models to the true measured values of the 2, 25, 50, 75 and $99 \%$ reflectance targets and used the model coefficients to transform the camera signal into reflectance and absorbance. These models were determined separately for each wavelength and image ROI column, except for the global transformations, which used wavelength specific mean values of the reference and target signals. The second image obtained of the 50\% reflectance target was then used as a test set for the 
determined transformations. Fig. 4B illustrates the reflectance spectra of a random test set pixel based on the different transformations. In addition, the true measured and interpolated values are given. The two classes of global and column-wise transformations showed clear differences in the reflectance values across the entire wavelength range. In addition, the global transformations led to significantly more noise especially towards the extremes of the wavelength range. The column-wise transformations considered the spatial dependency of the raw signal and resulted in significantly cleaner spectra. There was also a clear separation in magnitude between the two-point and the linear and quadratic column-wise transformations. Using the additional reflectance targets clearly improved the accuracy of the transformations within the wavelength range as could be expected.

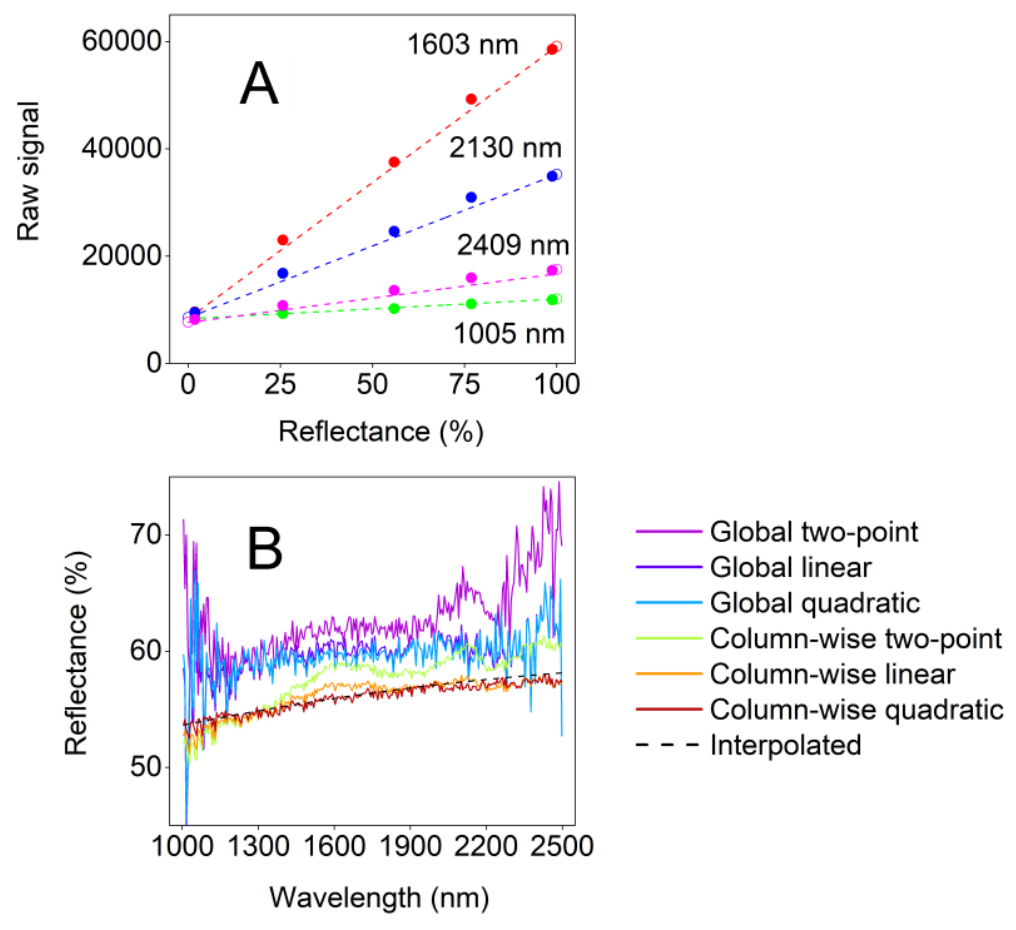

Fig. 4: Average raw signal values of the normalized white and example dark references and the reflectance targets in the middle of the imaging plane (A) and the reflectance spectra of a random test set pixel based on the determined reflectance transforms (B). The dashed lines in (A) illustrate a linear fit based on the normalized white reference and example dark current signals and in (B) the "true" measured and interpolated values of the 50\% reflectance target. 
The effects of the column-wise transformations on the 50\% test set ROI at $1603 \mathrm{~nm}$ are illustrated in Fig. 5. Results from the respective global transformations are provided in Fig. A.3 for brevity. Differences in the reflectance values of the individual image columns resulting from the class of global transformations could still be seen in Fig. A.2, which however disappeared with columnwise transformations (Fig. 5). As shown in Fig. 5, no clear spatial segregation was visible in the different column-wise transformations, however, the use of the linear and quadratic transformations decreased the differences between the $50 \%$ test set and true measured values at $1603 \mathrm{~nm}$. Fig. 5 also shows the spatial differences that were observed from the reflectance target images. In general, the effects of these spatial heterogeneities can be reduced by defocusing the images from the parts where the targets are situated [13]. In our case this was achieved by ensuring that there was a difference between the surface of the sample holder and the reflectance target in the focal plane.
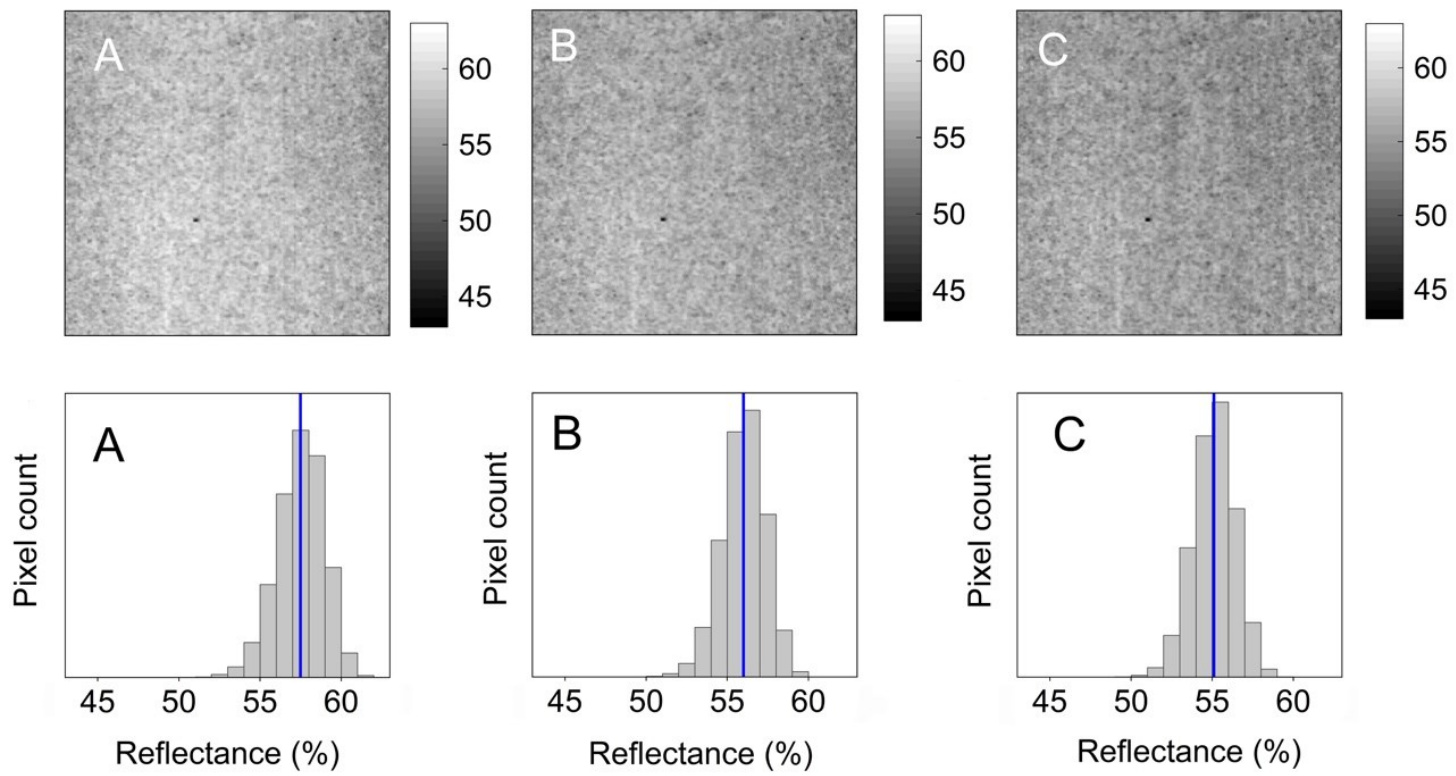

Fig. 5: The column-wise two-point (A), linear (B) and quadratic (C) transformations applied on the $50 \%$ test set image on $1603 \mathrm{~nm}$ (above) and the respective pixel histograms (below). The blue vertical lines in the histograms illustrate the average values across the $1603 \mathrm{~nm}$ image plane and the dashed red lines the respective "true" measured reflectance values. 
Bias and RMSE values of the reflectance test set are shown in Table 1. The parameters were determined by comparing with the wavelength-specific true reflectance values across all image pixels and wavelengths. Based on the results, the linear and quadratic transformations showed slightly higher negative bias compared with the two-point transformation in both classes and thus consistently resulted in lower reflectance than the true measured values. The class of global transformations however resulted in high RMSE values due to the variation in the reflectance spectra (Fig. 5 and Fig. A.2). The class of column-wise transformations significantly decreased the RMSE values based on the test set image. These trends can also be seen in Fig. 4B, where the variation in the pixel reflectance values decreased through the use of the column-wise transformations and the use of additional reflectance targets. Although the column-wise quadratic transformation led to slightly higher bias than the respective linear alternative, it showed the lowest RMSE (Table 1). This indicated that the column-wise quadratic transformation generated the lowest average measurement errors compared with the true measured values of the $50 \%$ reflectance test set. Previously Burger and Geladi [10] reported slightly lower bias values for the quadratic transformations using a staring imager on a 75\% reflectance target within 1100-1600 nm.

Table 1: Bias and RMSE values determined by comparing the $50 \%$ reflectance test set and the respective true reflectance values within 1000-2500 $\mathrm{nm}$.

\begin{tabular}{lllllll}
\hline Class & Global & & \multicolumn{3}{c}{ Column-wise } \\
Transformation & $\begin{array}{c}\text { Two- } \\
\text { point }\end{array}$ & Linear & Quadratic & $\begin{array}{l}\text { Two- } \\
\text { point }\end{array}$ & Linear & Quadratic \\
& & & & & & \\
\hline $\begin{array}{l}\text { Bias (\% } \\
\text { reflectance) }\end{array}$ & 0.45 & -0.82 & -0.92 & 0.41 & -0.84 & -0.93 \\
$\begin{array}{l}\text { RMSE (\% } \\
\text { reflectance) }\end{array}$ & 7.24 & 7.15 & 7.16 & 2.01 & 1.66 & 1.62 \\
& & & & & & \\
\hline
\end{tabular}




\subsection{Image regression}

After the $50 \%$ reflectance test set, the different reflectance transformations were applied to the raw signal images obtained from the prepared textile samples. The sample images were then converted to absorbance and eight average spectra were calculated from each ROI representing the chemically different areas in the calibration and test samples (Fig. 3). This translated to averaging across 2400 and 4800 pixel spectra and 48 image columns per each calibration and test spectrum, respectively. Example average spectra from the calibration sample ROI with 100\% PET are illustrated in Fig. 6. The results showed that the main differences within the average spectra were due to the two different classes of reflectance transformations. As illustrated in Fig. 6A, the column-wise quadratic reflectance transformation led to significantly cleaner absorbance spectra than the respective global alternative, which also showed significant variation within the different average spectra. As previously discussed, the global transformations used the wavelength specific global mean values of the white and dark references and the reflectance targets and neglected the respective spatial dependencies. Only small differences were seen between the column-wise two-point and quadratic reflectance transformations (Fig. 6B).

A

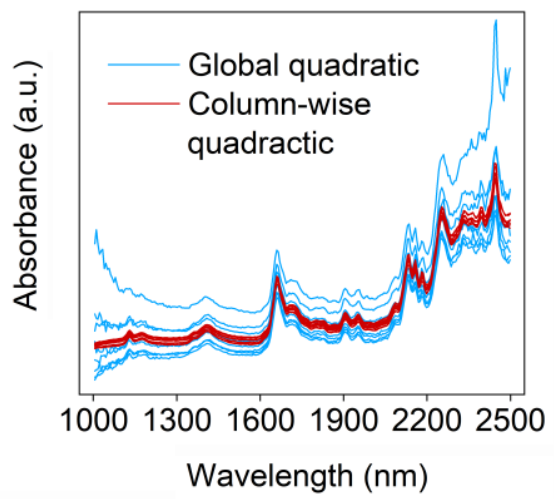

B

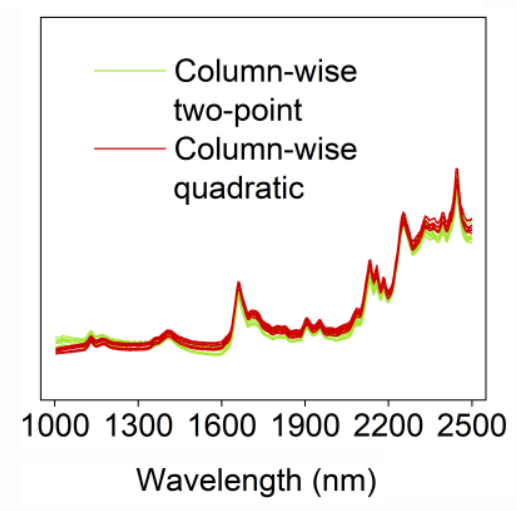

Fig. 6: Average calibration spectra for $100 \%$ PET based on the global and column-wise quadratic (A) and column-wise two-point and quadratic (B) transformations. 
The known PET contents of the image ROIs were then regressed against average calibration spectra obtained from the different reflectance transformations using PLS. The determined calibration and prediction diagnostics are illustrated in Fig. 7. Before calibration, the average spectra were preprocessed using standard normal variate $(\mathrm{SNV})$ transformation and mean centering. Spectral preprocessing is generally performed to remove unwanted measurement effects due to e.g. light scattering and to highlight differences in sample spectra for subsequent interpretation and is normal procedure in classical spectroscopy and spectral image analysis [27, 28]. However, preprocessing affects the calibration results. SNV transformation is a row-wise centering and scaling operation [29] that attempts to correct for scattering and sample illumination and is computationally fast especially when the same preprocessing procedure is to be applied to the individual pixel spectra of the sample images. As the objective of this work is to discuss the effect of reflectance transformations on image interpretation, we will limit the following discussion on SNV transformed and mean centered spectra.
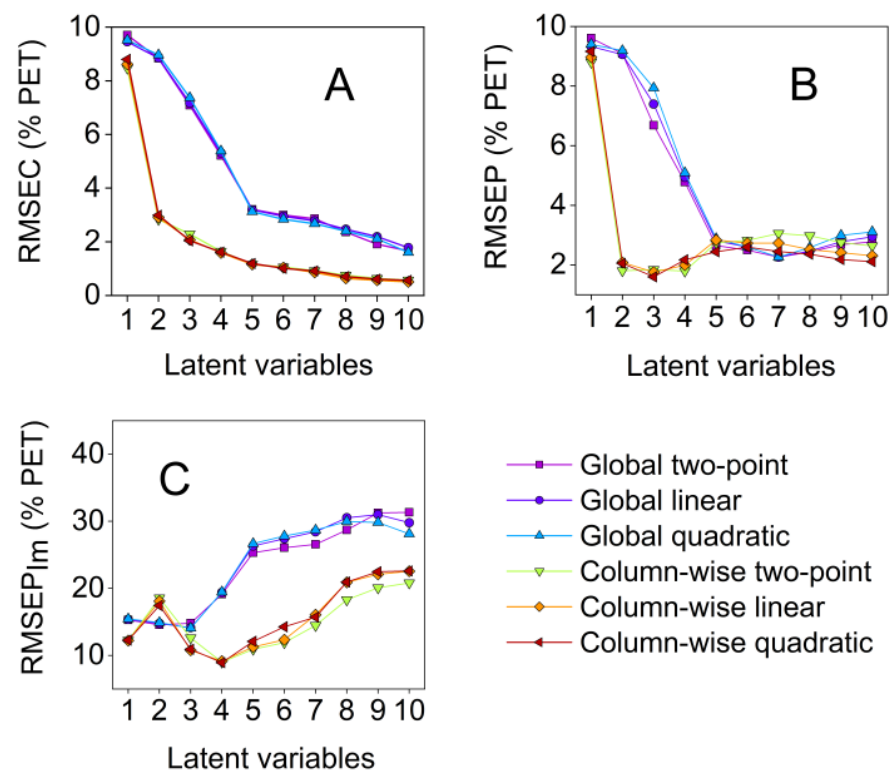

$$
\begin{aligned}
& \because \text { Global two-point } \\
& \because \text { Global linear } \\
& \square \text { Global quadratic } \\
& \square \text { Column-wise two-point } \\
& \because \text { Column-wise linear } \\
& \longleftarrow \text { Column-wise quadratic }
\end{aligned}
$$

Fig. 7: Calibration (A) and prediction errors based on extracted average spectra (B) and entire pixel distributions $(\mathrm{C})$ as a function of PLS latent variables for the different reflectance transforms. Spectral preprocessing was based on SNV transformation and mean centering. 
Calibration and prediction errors are often used as a tool to evaluate the correct number of latent variables and the pseudorank of a PLS model. The aim is to use as few latent variables as possible to maintain model robustness and simplicity while ruling out noise as overfitting generally leads to increased prediction errors. One way to estimate pseudorank is to look for shoulders or minima in average prediction errors expressed as RMSEP as a function of the number of latent variables. Based on the results, there was a clear difference in RMSEP between the two classes of reflectance transformations. As illustrated in Fig. 7B, the class of global reflectance transformations led to higher prediction errors based on extracted average spectra and did not show a minimum in RMSEP until seven latent variables were used. The required number of latent variables decreased with the use of column-wise reflectance transformations, which showed a minimum RMSEP with three or four latent variables. The column-wise two-point transformation suggested that 2-4 latent variables seemed appropriate with a minimum RMSEP of $1.79 \%$ PET with 4 latent variables. The clearest distinction in minimum RMSEP were however obtained with the column-wise linear and quadratic transformations, which led to minimum RMSEP's of $1.76 \%$ and $1.60 \%$ PET, respectively, with three latent variables. These results suggested that the use of additional reflectance targets improved the estimation of model pseudorank and decreased average prediction errors based on extracted average spectra.

Using calibration or prediction errors based on extracted average spectra is however slightly misleading in image regression as the spectra represent averages of image segments and not entire populations of individual pixel spectra. Gowen et al. [30] proposed the use of RMSEPIm to take into account the prediction residuals of individual image pixels. The authors showed that RMSEP $\mathrm{Im}$ enabled accurate pseudorank estimation from simulated images [30]. As illustrated by our results in Fig. 7C, RMSEP Im based on the global reflectance transformations showed a sharp increase after three latent variables, which indicated that choosing 7 latent variables based extracted average spectra and RMSEP (Fig. 7B) would in fact result in overfitting and unstable test set pixel 
predictions. The lowest prediction errors were obtained with the column-wise reflectance transformations using 4 latent variables (Fig. 7C). Although the differences in RMSEP Im within the class of column-wise transformations were small, the lowest average errors in predicting individual image pixels were obtained with the quadratic reflectance transformation (8.93\% PET) followed by the two-point (9.01\% PET) and linear alternatives (9.16\% PET).

The class of global reflectance transformations is generally not required if the references or reflectance targets cover the entire camera field of view or selected image scene. In other cases, however, the spatial dependencies between the target and sample segments are lost. Hyperspectral images obtained with a line-scanning instrument are generally constructed by scanning a row of pixels in the vertical column direction. The different reflectance transformations thus decrease measurement uncertainties and variation in raw signal intensity within the different image columns if the sample illumination and measurement conditions remain the same. Based on our results, the class of global reflectance transformations led to significantly higher average measurement errors based on the $50 \%$ reflectance test set. After converting the measured sample images into absorbance, the global transformations also decreased the quality of extracted average spectra, made it more difficult to reliable estimate PLS model pseudorank and led to higher average prediction errors based on extracted average spectra and individual test set pixels. These results are important as a convenient alternative in using additional reflectance targets is to place them internally within each sample image covering only part of the entire image scene. As illustrated by our findings, however, care needs to be taken to retain the spatial dependencies between the sample and reflectance target image columns to avoid decreasing image quality and the reliability of image interpretation.

Finally, the predicted PET contents in the test sample image based on the different column-wise transformations are illustrated in Fig. 8. The pixel-wise predictions were based on a PLS model with four latent variables as suggested by the global minimum in determined RMSEP Im (Fig. 7C). 
As shown in Fig. 8, it was difficult to separate the differences between the reflectance transformations based on the predicted concentration maps or respective histograms. However, for creating a more definitive comparison, we used the predicted pixel distributions based on previously defined test sample ROIs shown in Fig. 3B. Analyses of variance based on the ROIs showed that there was a statistically significant difference $(p$-values $<0.001)$ within the average predicted PET contents across all three ROIs (Table A.1). In all three cases, the difference between the average predicted and known PET contents decreased with the use of the additional reflectance targets. The closest agreement with the average predicted and known PET contents across all ROIs were obtained with the quadratic reflectance transformation (Table A.1). These results suggested that, although it was difficult to separate the effects of the different column-wise transformations based on the predicted concentration maps, the linear and quadratic transformations had an effect on the predicted pixel values and reduced average errors in the predicted concentration maps.
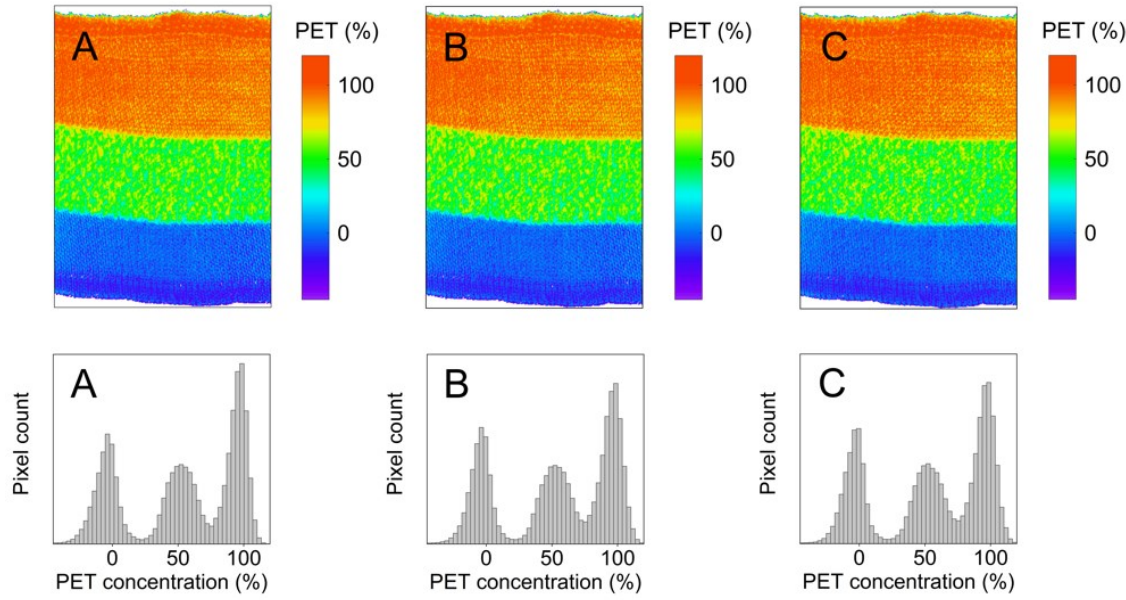

Fig. 8: Predicted PET concentrations (\%, mass basis) in the textile test sample based on the columnwise two-point (A), linear (B) and quadratic (C) reflectance transformations followed and hyperspectral image regression. The PLS calibration model was based on SNV transformed and mean centered spectra with four latent variables. Some outlier pixels are not shown in the histograms. 


\section{Conclusions}

Reflectance transformations govern the precision with which raw hyperspectral images are converted into reflectance in the intensity domain. For a line-scanning instrument, these transformations are best determined by considering the wavelength and column-wise specific spatial dependencies between the sample and target segments. Otherwise the spatial correlation between the segments is lost and only wavelength specific global mean values of the target signals have to be used. In this work we shown that the class of column-wise transformations, which were determined based on a series of externally calibrated reflectance targets, generated significantly cleaner spectra based on the $50 \%$ reflectance test set. The column-wise linear and quadratic transformations also decreased average measurement errors to $1.62-1.66 \%$ reflectance which was lower than the respective two-point alternative (2.01\% reflectance). During image interpretation based on PLS regression, the use of additional reflectance targets also made it easier to estimate model pseudorank and improved average prediction errors based on extracted average spectra and individual test image pixels. Comparing defined segments of the predicted concentration maps also showed that the column-wise quadratic transformation provided the closest agreement between average predicted and known PET contents in the test sample image. Based on these results, we recommend the use of additional reflectance targets for decreasing measurement uncertainties and improving image interpretation and the predicted concentration maps.

\section{Acknowledgements}

We acknowledge the contribution of Håkan Nilsson from the Swedish University of Agricultural Sciences in the practical arrangements for the imaging procedure and Anna Leinonen from Aalto University in the preparation of the textile samples. This work was financially supported by the Academy of Finland under grant agreement no. 309881 and the Strategic Research Council at the Academy of Finland under grant agreement no. 327296 - the FINIX project. 


\section{References}

[1] A.F.H. Goetz, Three decades of hyperspectral remote sensing of the Earth: a personal view, Remote Sens. Environ. 113 (2009) S5-S16.

[2] J. Burger, A. Gowen, Data handling in hyperspectral image analysis, Chemometrics Intell. Lab. Syst. 108 (2011) 13-22.

[3] P.J. Williams, S. Kucheryavskiy, Classification of maize kernels using NIR hyperspectral imaging, Food Chem. 209 (2016) 131-138.

[4] L.M. Kandpal, J. Tewari, N. Gopinathan, P. Boulas, B.-K. Cho, In-process control assay of pharmaceutical microtablets using hyperspectral imaging coupled with multivariate analysis, Anal. Chem. 88 (2016) 11055-11061.

[5] D. Nouri, Y. Lucas, S. Treuillet, Hyperspectral interventional imaging for enhanced tissue visualization and discrimination combining band selection methods, Int. J. Comput. Assist. Radiol. Surg. 11 (2016) 2185-2197.

[6] G. Mirschel, O. Daikos, T. Scherzer, C. Steckert, Near-infrared chemical imaging used for inline analysis of inside adhesive layers in textile laminates, Anal. Chim. Acta 932 (2016) 69-79.

[7] J. Pan, T.-G. Cha, F. Li, H. Chen, N.A. Bragg, J.H. Choi, Visible/near-infrared subdiffraction imaging reveals the stochastic nature of DNA walkers, Sci. Adv. 3 (2017) e1601600.

[8] B.G. Osborne, R. Fearn, P.H. Hindle, Practical NIR Spectroscopy with applications in Food and Beverage Analysis, 2nd ed., Pearson Education Limited, United Kingdom, 1993.

[9] A. de Juan, S. Piqueras, M. Maeder, T. Hancewicz, L. Duponcel, R. Tauler, Chemometric Tools for Image Analysis, in: R. Salzer, H.W. Siesler (Eds.), Infrared and Raman Spectroscopic Imaging, Wiley-VCH Verlag GmbH \& Co, Weinheim, 2014, pp. 57-110.

[10] J. Burger, P. Geladi, Hyperspectral NIR image regression part I: calibration and correction, J. Chemometr. 19 (2005) 355-363.

[11] P. Geladi, J. Burger, T. Lestander, Hyperspectral imaging: calibration solutions and problems, Chemometrics Intell. Lab. Syst. 72 (2004) 209-217. 
[12] M. Manley, Near-infrared spectroscopy and hyperspectral imaging: non-destructive analysis of biological materials, Chem. Soc. Rev. 43 (2014) 8200-8214.

[13] J. Burger, Hyperspectral NIR Image Analysis - Data Exploration, Correction and Regression, Unit of Biomass Technology and Chemistry, Swedish University of Agricultural Sciences, Umeå, Sweden, 2006.

[14] G. Polder, G. van der Heijden, P. Keizer, I. Young, Calibration and characterisation of imaging spectrographs, J. Near Infrared Spectrosc. 11 (2003) 193-210.

[15] M. Bürmen, F. Pernuš, B. Likar, Spectral characterization of near-infrared acousto-optic tunable filter (AOTF) hyperspectral imaging systems using standard calibration materials, Appl. Spectrosc. 65 (2011) 393-401.

[16] B. Boldrini, W. Kessler, K. Rebner, R.W. Kessler, Hyperspectral imaging: a review of best practice, performance and pitfalls for in-line and on-line applications, J. Near Infrared Spectrosc. 20 (2012) 483-508.

[17] J. Burger, P. Geladi, Hyperspectral NIR image regression part II: dataset preprocessing diagnostics, J. Chemometr. 20 (2006) 106-119.

[18] C. Blanch-Perez-del-Notario, W. Saeys, A. Lambrechts, Hyperspectral imaging for textile sorting in thge visible-near infrared range, Journal of Spectral Imaging 8 (2019) a17.

[19] G. Mirschel, O. Daikos, T. Scherzer, C. Steckert, Near-infrared chemical imaging used for inline analysis of functional finishes on textiles, Talanta 188 (2018) 91-98.

[20] A. Sluiter, B. Hames, R. Ruiz, C. Scarlata, J. Sluiter, D. Templeton, D. Crocker, Determination of Structural Carbohydrates and Lignin in Biomass, Laboratory Analytical Procedure (LAP), National Renewable Energy Laboratory, Golden, Colorado, 2011.

[21] Specim, Spectral Imaging Ltd., 2019. http://www.specim.fi/hyperspectral-cameras/. (Accessed July 25th 2019).

[22] T. Næs, T. Isaksson, T. Fearn, T. Davies, A user-friendly guide to Multivariate Calibration and Classification, IM Publications, Chichester, 2017. 
[23] P. Geladi, H. Grahn, M. Manley, Data Analysis and Chemometrics for Hyperspectral Imaging, in: S. Šašic, Y. Ozaki (Eds.), Raman, Infrared, and Near-Infrared Chemical Imaging, John Wiley \& Sons, Inc., Hoboken, 2010, pp. 93-107.

[24] P. Geladi, B. Kowalski, Partial least-squares regression: a tutorial, Anal. Chim. Acta 185 (1986) 1-17.

[25] H. Martens, T. Næs, Multivariate Calibration, John Wiley \& Sons Ltd, New York, 1989.

[26] S. Wold, M. Sjöström, L. Eriksson, PLS-regression: a basic tool of chemometrics, Chemometrics Intell. Lab. Syst. 58 (2001) 109-130.

[27] J.M. Amigo, H. Babamoradi, S. Elcoroaristizabal, Hyperspectral image analysis. A tutorial, Anal. Chim. Acta 896 (2015) 34-51.

[28] Å. Rinnan, Pre-processing in vibrational spectroscopy - when, why and how, Anal. Methods 6 (2014) 7124-7129.

[29] R. Bro, A.K. Smilde, Centering and scaling in component analysis, J. Chemometr. 17 (2003) 16-33.

[30] A. Gowen, J. Burger, C. Esquerre, G. Downey, C. O’Donell, Near infrared hyperspectral image regression: on the use of prediction maps as a tool for detecting model overfitting, J. Near Infrared Spectrosc. 22 (2014) 261-270.

[31] K.H. Esbensen, P. Geladi, Principles of proper validation: use and abuse of resampling for validation, J. Chemometr. 24 (2010) 168-187. 